\title{
Identification of cycles in sunspot numbers using percentage variance technique

\author{
Iwok, Iberedem A.
}

\author{
Department of Mathematics/Statistics, Faculty of Science, \\ University of Port Harcourt, P.M.B. 5323 Port Harcourt, Rivers State, Nigeria
}

\begin{abstract}
In this paper, a method of percentage variance was used to identify the cycle in sunspot numbers $\left(X_{t}\right)$ given the assumption that the cycle is not known a priori. The idea here was to estimate the percentage of the variance accounted for by each of the set of the different sinusoids using Fourier coefficients of the original time series $\left(X_{t}\right)$. Remarkably, the $10^{\text {th }}$ harmonic explains the greatest percentage of the overall variance that corresponds to the highest amplitude with a dominant cycle of $10.8 \approx 11$ years.
\end{abstract}

Keywords: Fourier coefficients, Amplitudes, Harmonics, Periodicities.

\section{INTRODUCTION}

The sunspot series arises from the fact if one looks at the surface of the sun; it will usually contain dark spots which are areas of relatively coolness. In 1610, shortly after viewing the sun with his new telescope, Galileo made the first European observations of sunspots. Continuous daily observations started at the Zurich Observatory in 1849 and earlier observations have been used to extend the records back to 1610(Hathaway, 2010). Since the mid eighteen century, an observatory in Switzerland has been recording monthly, a measure of the number of sunspots, for reasons of their own and presumably at considerable risk to the austronomer's eyesight, thus providing one of the longest time series available for analysis (Granger, 1989).

The fact that the sunspot measure reaches a peak roughly every eleven years for reasons that are still not completely understood, leads to a cycle in the data. Being cyclic, Granger (1989) proposed a model for such series to be of the form:

$X_{t}=A \cos (\omega t+\theta)+\varepsilon_{t}$

Where $\varepsilon_{t}$ is a white noise series and the first term is a purely periodic component with amplitude $A$ and frequency $\omega$ and $\theta$ is the phase value. However, when such models were fitted to the series, a worth while fit was usually obtained, but the estimated residuals were frequently observed to still be too smooth to be white noise.

Rogers and Richards (2004), analysed archival data on sunspot numbers and sunspot areas to derive the solar activity cycles based on these variables. From their work, there were able to identify 11-year
Schwabe cycle, Hale cycle, Gleissberg cycle and a cycle at approximately 10 years.

$\mathrm{Xu}$ et al (2010) applied the empirical mode description and autoregressive model to long term sunspot numbers. The method was evaluated using data of the solar 23; and the result was remarkably the predictions made by the solar dynamo and precursor approaches for cycle 23. The 11 year cycle of the number of sunspots was first demonstrated by Henrich Schwab in 1843.

Richard (2005) also confirmed the existence of cycles in sunspot numbers using early historical records, and this was calculated to be 11.1 years. By Richard's calculation, it means that sunspot numbers rise and fall approximately every 11.1 years. As noted, most earlier works on determining the number of cycles of a periodic series were based either on examining the plot of the time series or from past researches.

When the cycle is not known a priori; either because there is little or no past research or because the cycles are not regular enough to be identified easily from visual examination of a graph of the time series; then other exploratory methods are needed to identify the cycle of the series.

The major problem in periodic analysis is the determination of the appropriate cycle length of the series. When once the cycle or period is identified, the series can thus be modeled using Ordinary least squares (Bloomfield, 1976).

Having noted the major problem to be that of cycle identification, this work seeks to determine the cycle 
length of the sunspot numbers using Percentage Variance technique.

Method of Analysis: The method of analysis to be used here is the percentage Variance technique (PVT). PVT estimates the percentage of the variance in the time series that is accounted for by each of a set of different sinusoids. The method makes use of the Fourier coefficient of the original series $\left(X_{t}\right)$. Thus we assume that $X_{t}$ can be expressed as the algebraic sum of a series of simple sine and cosine curves with amplitudes $\left(A_{i}\right)$ and frequencies $\left(f_{i}\right)$. Thus we have:

$X_{t}=\bar{X}+\sum_{i=1}^{q}\left(a_{i} \cos \omega_{i} t+b_{i} \sin \omega_{i} t\right)$

Where $a_{i}^{\prime} s$ and $b_{i}{ }^{\prime} s$ are the Fourier coefficients for the sine and cosine terms respectively and are given by

$$
\begin{gathered}
a_{i}=\frac{2}{N} \sum X_{t} \cos \omega_{i} t \quad ; \quad b_{i}=\frac{2}{N} \sum X_{t} \sin \omega_{i} t \\
\omega_{i}=2 \pi f_{i} ; f_{i}=\frac{i}{N} ; q=\frac{N}{2} \text { if } N \text { is even ; } q=\frac{N-1}{2}
\end{gathered}
$$

if $N$ is odd ; $i$ is any harmonic (or cycle) ; $N$ is the series length ; $\bar{X}$ is the mean about which the series fluctuates.

Table 1: Tabulated Result of the Fourier Analysis

\begin{tabular}{|l|l|l|l|l|l|l|l|}
\hline Harmonics & Periodicity & Amplitude & \%Variance & Harmonics & Periodicity & Amplitude & \%Variance \\
\hline 1 & 108.00 & 154.39 & 6.34 & 28 & 3.86 & 1.19 & 0.00 \\
\hline 2 & 54.00 & 36.81 & 0.30 & 29 & 3.72 & 0.23 & 0.00 \\
\hline 3 & 36.00 & 24.60 & 0.12 & 30 & 3.60 & 1.49 & 0.00 \\
\hline 4 & 27.00 & 99.30 & 1.67 & 31 & 3.48 & 2.59 & 0.00 \\
\hline 5 & 21.6 & 26.95 & 0.16 & 32 & 3.36 & 0.84 & 0.00 \\
\hline 6 & 18.00 & 132.01 & 3.85 & 33 & 3.27 & 3.15 & 0.00 \\
\hline 7 & 15.43 & 138.05 & 4.12 & 34 & 3.18 & 2.48 & 0.00 \\
\hline 8 & 13.50 & 166.00 & 7.05 & 35 & 3.09 & 0.52 & 0.00 \\
\hline 9 & 12.00 & 212.18 & 9.94 & 36 & 3.00 & 0.57 & 0.00 \\
\hline 10 & 10.8 & 3076.02 & 34.35 & 37 & 2.92 & 0.94 & 0.00 \\
\hline 11 & 9.82 & 219.07 & 9.99 & 38 & 2.84 & 0.95 & 0.00 \\
\hline 12 & 9.00 & 145.38 & 4.67 & 39 & 2.77 & 1.17 & 0.00 \\
\hline 13 & 8.31 & 151.01 & 4.91 & 40 & 2.70 & 0.97 & 0.00 \\
\hline 14 & 7.71 & 224.01 & 11.08 & 41 & 2.63 & 1.69 & 0.00 \\
\hline 15 & 7.20 & 28.03 & 0.17 & 42 & 2.57 & 0.62 & 0.00 \\
\hline 16 & 6.75 & 30.00 & 0.20 & 43 & 2.51 & 1.30 & 0.00 \\
\hline 17 & 6.35 & 2.97 & 0.00 & 44 & 2.45 & 2.29 & 0.00 \\
\hline 18 & 6.00 & 9.34 & 0.02 & 45 & 2.40 & 2.69 & 0.00 \\
\hline 19 & 5.68 & 6.39 & 0.01 & 46 & 2.35 & 1.33 & 0.00 \\
\hline 20 & 5.40 & 4.86 & 0.01 & 47 & 2.30 & 1.26 & 0.00 \\
\hline 21 & 5.41 & 6.54 & 0.01 & 48 & 2.25 & 0.22 & 0.00 \\
\hline 22 & 4.91 & 6.54 & 0.01 & 49 & 2.20 & 1.36 & 0.00 \\
\hline 23 & 4.70 & 1.92 & 0.00 & 50 & 2.16 & 2.09 & 0.00 \\
\hline 24 & 4.50 & 1.35 & 0.00 & 51 & 2.12 & 0.82 & 0.00 \\
\hline 25 & 4.32 & 1.42 & 0.00 & 52 & 2.08 & 1.07 & 0.00 \\
\hline 26 & 4.15 & 1.27 & 0.00 & 53 & 2.04 & 1.96 & 0.00 \\
\hline 27 & 4.00 & 1.90 & 0.00 & 54 & 2.00 & 1.15 & 0.00 \\
\hline
\end{tabular}

The importance of any harmonic is a function of the size of a parameter called its amplitude. number of harmonics that can be extracted is 54 (that is $\frac{N}{2}$ ). This is because after $\frac{N}{2}$ harmonics, the values wrap around and begin to duplicate themselves. The percentage variance $(\% V)$ explained by any harmonic or cycle is thus given by $\% V_{i}=\frac{A_{i}^{2}}{2 \sigma_{X}^{2}} \times 100 ;$ where $\sigma_{X}^{2}$ is the variance of $X_{t}$.

The idea here is to identify which, if any periodic component explains a large enough percentage of the variance in the time series of interest and to describe their cycle lengths and amplitudes in situations where the periods of cycles are not known a priori.

Data Analysis, Result and Interpretation: Annual averaged sunspot numbers [(1900-2007), see table 2] was used for the study. The analysis was performed with the aid of software called Minitab. Given the different harmonics ( $i=1,2, \ldots, 54)$; the periodicities, amplitude and percentage Variance are displayed in the table 1 below:
The amplitude ${ }_{A_{i}=\sqrt{a_{i}^{2}+b_{i}^{2}}}$. For this work $(N=108)$, the 
Since $N=108 ; 54$ harmonics are extracted. The $10^{\text {th }}$ harmonic explains the greatest percentage of the overall variance and also has the highest amplitude. The periodicity indicated by this harmonic is $N / i=108 / 10=10.8 \approx 11$ years. We thus conclude that the dominant movement in the sunspot data is a cycle with a periodicity of 11 years.

Table 2 : Annual Averaged Wolfer's Sunspot Numbers (1900-2007) Courtesy: Solar Index Data Centre

\begin{tabular}{|l|l|l|l|l|l|l|l|l|l|l|l|}
\hline YEAR & & YEAR & VALUE & YEAR & VALUE & YEAR & VALUE & YEAR & VALUE & YEAR & VALUE \\
\hline 1900 & 9.5 & 1918 & 80.6 & 1936 & 79.7 & 1954 & 4.4 & 1972 & 68.9 & 1990 & 142.6 \\
\hline 1901 & 2.7 & 1919 & 63.6 & 1937 & 114.4 & 1955 & 38 & 1973 & 38 & 1991 & 145.7 \\
\hline 1902 & 5 & 1920 & 37.6 & 1938 & 109.6 & 1956 & 141.7 & 1974 & 34.5 & 1992 & 94.3 \\
\hline 1903 & 24.4 & 1921 & 26.1 & 1939 & 88.8 & 1957 & 190.2 & 1975 & 15.5 & 1993 & 54.6 \\
\hline 1904 & 42 & 1922 & 14.2 & 1940 & 67.8 & 1958 & 184.8 & 1976 & 12.6 & 1994 & 29.9 \\
\hline 1905 & 63.5 & 1923 & 5.8 & 1941 & 47.5 & 1959 & 159 & 1977 & 27.5 & 1995 & 17.5 \\
\hline 1906 & 53.8 & 1924 & 16.7 & 1942 & 30.6 & 1960 & 112.3 & 1978 & 92.5 & 1996 & 8.6 \\
\hline 1907 & 62 & 1925 & 44.3 & 1943 & 16.3 & 1961 & 53.9 & 1979 & 155.4 & 1997 & 21.5 \\
\hline 1908 & 48.5 & 1926 & 63.9 & 1944 & 9.6 & 1962 & 37.6 & 1980 & 154.6 & 1998 & 64.3 \\
\hline 1909 & 43.9 & 1927 & 69 & 1945 & 33.2 & 1963 & 27.9 & 1981 & 140.4 & 1999 & 93.3 \\
\hline 1910 & 18.6 & 1928 & 77.8 & 1946 & 92.6 & 1964 & 10.2 & 1982 & 115.9 & 2000 & 199.6 \\
\hline 1911 & 5.7 & 1929 & 64.9 & 1947 & 151.6 & 1965 & 15.1 & 1983 & 66.6 & 2001 & 111 \\
\hline 1912 & 3.6 & 1930 & 35.7 & 1948 & 136.3 & 1966 & 47 & 1984 & 45.9 & 2002 & 104 \\
\hline 1913 & 1.4 & 1931 & 21.2 & 1949 & 134.7 & 1967 & 93.8 & 1985 & 17.9 & 2003 & 63.7 \\
\hline 1914 & 9.6 & 1932 & 11.1 & 1950 & 83.9 & 1968 & 105.9 & 1986 & 13.4 & 2004 & 40.4 \\
\hline 1915 & 47.4 & 1933 & 5.7 & 1951 & 69.4 & 1969 & 105.5 & 1987 & 29.4 & 2005 & 29.8 \\
\hline 1916 & 57.1 & 1934 & 8.7 & 1952 & 31.5 & 1970 & 104.5 & 1988 & 100.2 & 2006 & 15.2 \\
\hline 1917 & 103.9 & 1935 & 36.1 & 1953 & 13.9 & 1971 & 66.6 & 1989 & 157.6 & 2007 & 7.55 \\
\hline
\end{tabular}

\section{CONCLUSION:}

The basic measure of the solar activity is the number of the sunspot visible on the solar disk at any given time. The more the sunspot, the more active is the sun(Sharaf,2009). Due to its enormous importance in climatology, sunspot analysis has attracted huge attention in academic and other sectors of life.

This work has analysed the sunspot data using percentage variance technique. The result obtained showed a dominant cycle of $10.8 \approx 11$ years. In other words, this cycle repeats itself every 11 years, which agrees with the results of the earlier works of the afformentioned authors in the review.

\section{REFERENCES}

Bloomfield, P. (1976). Fourier analysis of time series: An introduction. New York, Wiley
Granger, C.W.J. (1989). Forcasting in business and economics(second edition). Academic press inc; Harcourt brace. Jovanovich publishers. Boston, New York.

Hathaway, D. H. (2010). Update of sunspot cycle. National aeronautics and space administration. Marshall flight space centre. Hathaway @ nasa.gov

Richard, H.T.(2005). Time series analysis of the sunspot cycle. Indian Journal of applied statistics.Vol.3, No.15, pp 46-55.

Rogers, M.L and Richards, M.T. (2004). Periodogram analysis of sunspot numbers and areas. Bulletin of the American astronomical society. Vol.8, No.2.

$\mathrm{Xu}, \mathrm{T}$. et al (2010). Long term sunspot number prediction based on EMD analysis and AR model. Chinese journal of astronomy and astrophysics. Vol.8, No. 337. 